\title{
Autogestión hospitalaria en red I: Competencias esenciales para la gestión
}

\author{
MARCOS VERGARA $^{(1)}$ y LIGIA BISAMA $^{(2)}$
}

\section{RESUMEN}

Este documento hace referencia al Programa de Acompañamiento de Hospitales que la Subsecretaría de Redes Asistenciales del Ministerio de Salud de Chile impulsó para la autogestión hospitalaria en red. Dice relación con el programa de la Escuela de Salud Pública de la Universidad de Chile, diseñado para facilitar el desarrollo de tres competencias esenciales, que los hospitales requerirían para la implementación de una política pública con enfoque de derechos (el Plan Auge), en un escenario competitivo. A partir de los elementos centrales de la reforma sectorial y del marco teórico utilizado, se definieron dichas competencias: desarrollo de la cartera de servicios, gestión de la referencia y contrareferencia de pacientes y gestión de las listas de espera.

\section{ANTECEDENTES DE LA AUTOGESTIÓN HOSPITALARIA}

\section{Legislación para la Reforma del sector de la salud en Chile}

Quien asumió como Presidente de la República a partir del 2000, anunció que el sector de la salud sería objeto de reformas. Lo inspiraban objetivos sanitarios y de equidad en el acceso, en la calidad de los servicios provistos y en la protección financiera brindada a las personas, en una perspectiva de protección universal.
Entre los años 2000 y 2005, un Comité Interministerial (Hacienda, Salud, Trabajo y Secretaría General de la Presidencia) estuvo a cargo de un proceso que culminó en leyes que incluyeron los contenidos de la reforma. Se creó una Secretaría Ejecutiva en el Ministerio de Salud para su diseño y traducción en los proyectos de ley que se enviaron a tramitación ${ }^{1}$.

La Ley N¹9.888 del 2003, "de Financiamiento", consideró alzas del Impuesto al Valor Agregado y de otros impuestos específicos para financiar el Régimen de Garantías Explicitas de Salud (Plan Auge) y otros programas.

La Ley $N^{\circ} 19.895$ del 2003, "Corta de Isapres", instruyó sobre normas de solvencia financiera para las Instituciones de Salud Previsional y sobre las transferencias de cartera entre éstas y resolvió el destino de la cartera de beneficiarios de la Isapre de Inverlink, que había quebrado.

La Ley N¹9.937 de 2004, “de Autoridad Sanitaria”, separó las funciones de salud pública de las de prestación de servicios, lo que significó la creación de dos subsecretarías -de Salud Pública y de Redes Asistenciales- y a nivel territorial, el traspaso de las funciones de salud pública desde los servicios de salud a las Secretarías Regionales Ministeriales. Al interior de los servicios de salud, definidos como "gestores de redes", la ley creó la figura de los hospitales autogestionados en red. Por último, la Ley amplió las facultades de la Superintendencia de Isapres asignándole la

\footnotetext{
(1) Escuela de Salud Pública. Facultad de Medicina. Universidad de Chile. Independencia 939. Santiago. Chile. mvergara@med.uchile.cl.

(2) Emerge Consultores. Santiago. Chile.
} 
fiscalización de Fonasa y la regulación de los prestadores de salud para la garantía de calidad del Plan Auge.

La Ley N¹9.966 del 2004, "del Plan Auge", estableció un plan de salud obligatorio para Fonasa e Isapres (Régimen de Garantías Explicitas o Plan Auge), consistente en confirmación diagnóstica y tratamientos estandarizados para un conjunto de enfermedades priorizadas por su alto impacto sanitario y social. Este es el "core" de la Reforma y su característica clave es la exigibilidad de derechos, en términos de acceso, oportunidad, calidad y cobertura financiera de las prestaciones.

La Ley $\mathrm{N}^{\circ} 20.015$ del 2005, "Larga de Isapres", regula la adecuación anual de contratos, alzas de precios y tablas de factores de riesgo por edad y sexo y establece Fondo de Compensación para el Plan Auge, que implica transferencias de recursos entre las Isapres, basadas en diferenciales de riesgo de sus carteras de afiliados.

\section{Reforma de Salud con enfoque de derecho}

La idea de políticas públicas con enfoque de derechos converge con la Reforma durante su diseño, si bien data desde hace unos 15 años al menos. No hubo en el discurso del Presidente o de otros voceros de la Reforma, una alusión directa al concepto, no obstante el Plan Auge corresponde a una política con "enfoque de derechos".

Esta idea ${ }^{2}$ se funda en la reflexión acerca de los derechos humanos y en los giros acaecidos en los programas del Banco Mundial, promotores de reformas de segunda generación. Se reduce el conflicto entre universalización y focalización, aceptándose la idea de que la redistribución de recursos es necesaria para garantizar derechos a quienes no los tienen, en un contexto de universalización.

La universalización de derechos supone dos requisitos: i) fuerza de exigibilidad, es decir, mecanismos a través de los cuales los derechos pueden ser ejercidos $\mathrm{y}$, ii) alta cuota de "accountability" en los organismos involucrados, para garantizar el cumplimiento de los derechos.

De lo anterior derivan, en primer lugar, requerimientos de cambio institucional, si se considera que muchos organismos toman contacto por primera vez con este tipo de políticas. En segundo lugar, necesidad de contar con información para "garantizar las garantías". Y, por último, derechos idénticos ${ }^{3}$ para todos no políticas "ad-hoc" para los pobres- asunto especialmente desafiante en la actualidad en Chile.

\section{Enfoque de derechos y gestión hospitalaria}

La práctica de una política pública con enfoque de derechos en salud está determinada por la gestión de los hospitales públicos. Lo anterior tiene que ver con la presencia de esquemas de gobierno corporativo indeterminados y vulnerables a la "captura" por parte de interesados, así como con marcos jurídico-administrativos estrechamente vinculados a las rígidas reglas que regulan la administración del Estado -como el Estatuto Administrativo- y las cuestiones propias de la política fiscal. Esta es la primera idea que cabe establecer ${ }^{4}$.

La política fiscal impone sobre los hospitales el rigor de presupuestos con reglas estrictas de expansión y, en ese marco, el control de la dotación de personal y de la escala única de sueldos son básicos para contener el gasto. La deuda con proveedores de los hospitales -que se resuelve tradicionalmente con aportes estatales extraordinarios-, así como las listas de espera que resultan de la restricción de oferta y de otros trastornos funcionales de la Red, son expresión de las limitaciones que existen para operar.

Los chequeos realizados por la Superintendencia de Salud detectan incumplimientos de las garantías del Plan Auge a beneficiarios Fonasa. No obstante el Plan Auge ha significado una transformación del perfil productivo de los hospitales, el cumplimiento de las garantías se encuentra con una restricción de orden semi-estructural, que combina falta de recursos con limitaciones para gestionar.

Segunda idea: la oferta de servicios del sector privado de salud en Chile es importante en tamaño, diversidad y complejidad técnica y en 
la última década duplicó su capacidad instalada ${ }^{5}$, invirtiendo muy por encima de lo observado en el sector público. Esto implica que: i) hay un relativo déficit de infraestructura y tecnología en los hospitales públicos y, ii) el sector privado constituye una alternativa de atención.

En síntesis, el ambiente es competitivo 6 , particularmente en un escenario con derechos exigibles, dadas las limitaciones en la capacidad de cumplimiento de las garantías vía hospitales públicos. Estas harán necesario recurrir a prestadores privados para cumplirlas, prestadores que no presentan problemas de expansión de oferta, captando incluso recursos profesionales de los propios hospitales públicos y profundizando su crisis.

Una de las cuestiones que la Reforma consideró fue la denominada Autogestión Hospitalaria, consistente en la transferencia de facultades administrativas de los servicios de salud a los hospitales ${ }^{7}$. Sin embargo, desde un comienzo se habló de "autogestión en red", lo que agrega una tercera idea a la discusión.

La "autogestión en red" implica introducir racionalidad en el uso de servicios en distintos niveles de capacidad resolutiva ${ }^{8}$ y se ponen en juego capacidades de los hospitales para definir y administrar su cartera de servicios -aquello que los hospitales están en condiciones de ofrecer-, mecanismos de referencia y contrareferencia de pacientes y, finalmente, sus listas de espera. Estas capacidades son esenciales en un hospital público autogestionado en red.

\section{PROGRAMA DE ACOMPAÑAMIENTO PARA LA AUTOGESTIÓN HOSPITALARIA EN RED}

La Ley de Autoridad Sanitaria estableció una lista de 59 hospitales que podrían postular a la categoría de Autogestionados en Red (EAR). Entre los años 2005 y 2007, once cumplieron con las condiciones establecidas en el instrumento de evaluación. De acuerdo a lo establecido en la Ley, los hospitales restantes entrarían en régimen EAR a partir del 1 de enero del 2009.
Antes del cumplimiento del plazo, el Ministerio de Salud declaró que los hospitales no se encontraban preparados para enfrentar la condición de EAR y convino con el Parlamento el aplazamiento de un año para la entrada en vigencia de esta disposición. Simultáneamente, se comprometió la realización de un programa de acompañamiento para esos hospitales.

Siguiendo el compromiso establecido con el Parlamento 9 , el Ministerio desarrolló un programa de acompañamiento de hospitales y convocó a desarrollar propuestas de trabajo en ese marco $^{10}$. El programa puso foco en los hospitales rezagados y distribuyó tipos de acompañamiento de acuerdo a los perfiles de necesidades diagnosticados, uno de los cuales se orientó a la autogestión hospitalaria en red, objeto de esta reflexión.

\section{MARCO TEÓRICO: DESARROLLO DE COMPETENCIAS PARA LA AGREGACIÓN DE VALOR}

Las estrategias organizacionales permiten establecer cursos de acción y producir cooperación alrededor de directrices comunes, conciliando fuerzas para la estabilidad y el cambio. Esto implica que los directivos formulen la estrategia y se encarguen de su implementación, pero también generen condiciones que permitan que aquella surja de la práctica organizacional ${ }^{11}$. Para ello es importante asegurar que la estrategia se despliegue, destacándose los aportes de Kaplan y Norton, que incorporan el uso de Mapas Estratégicos ${ }^{12}$.

El valor de las organizaciones se relaciona con los beneficios que reportan a sus grupos de interés a través del tiempo. Pero las organizaciones deben saber cómo generar ese valor, dado que enfrentan contextos más exigentes. En consecuencia, mantener y acrecentar el valor de una organización requiere de un proceso de aprendizaje organizacional constante, entendido como "el proceso de creación, distribución, uso e intercambio de conocimientos que tiene como propósito agregar 
valor a la organización"13. Este hecho conduce a la necesidad de entender muy bien cómo generar valor y cómo se producen los aprendizajes que lo hacen posible.

Para generar valor las organizaciones deben identificar sus capacidades o competencias esenciales -core competences-. Las competencias de una organización son "un conjunto de calificaciones y tecnologías", mientras que una competencia esencial "representa la suma de conocimientos incorporados a diversos conjuntos de calificaciones y unidades organizativas..., (siendo) muy improbable que una competencia esencial resida en una sola persona o en un pequeño equipo" ${ }^{14}$. Bajo este concepto, Hamel y Prahalad ${ }^{15}$ distinguieron aquellas capacidades organizacionales relativamente estables que cumplen con los siguientes requisitos: a) proveer acceso potencial a una variedad de mercados, b) contribuir a los beneficios percibidos por el consumidor en el producto final y, c) ser difíciles de imitar.

Le Boterf ${ }^{16}$ se refiere de la siguiente manera a una competencia esencial: a) estrategia, necesaria para realizar las orientaciones estratégicas, b) competitividad, requerida para obtener o mantener una ventaja competitiva $\mathrm{y}, \mathrm{c}$ ) especificidad, una construcción específica de la organización que no puede ser trasportada a otras.

Cuando se han establecido cuáles son las competencias esenciales, es posible alinear en torno a ellas las competencias clave de cada una de las áreas de la organización y de los equipos de trabajo. Los equipos de trabajo, de hecho, son un medio para obtener competencias colectivas.

\section{LAS "CORE COMPETENCES" PARA LA AUTOGESTIÓN DE HOSPITALES PÚBLICOS EN RED}

Las tareas propias del gestor de red se encuentran en definición, a partir de la transferencia de las funciones de salud pública a las SEREMI y de la transferencia de facultades administrativas de los servicios de salud hacia los hospitales.
El foco de este trabajo está puesto en la gestión "en red" desde la perspectiva del hospital y no desde la perspectiva del servicio de salud. Conceptualmente, se entiende que la gestión "en red" y la autogestión hospitalaria son fenómenos complementarios e interdependientes ${ }^{17}$. En la intersección de estos dos elementos surgen las competencias esenciales para un hospital autogestionado que opera en red. Estas son competencias esenciales propias de un hospital que forma parte de una red de atención "cerrada" y están a la base del cumplimiento de los derechos exigibles de que son titulares los beneficiarios de Fonasa, determinando aquello la gran estrategia organizacional (Figura 1).

Aplicando a los hospitales públicos los tres criterios que establecieron Hamel y Prahalad para identificar las competencias esenciales, ellas debieran: a) mantener el potencial de mercado actual, impidiendo la transferencia de valor a los prestadores privados mediante la satisfacción de los usuarios, b) dar la atención requerida a los usuarios dentro de los plazos que establecen las garantías y, c) distinguirse como oferta pública, lo que se cumple en la medida que las competencias no sean aspectos que los prestadores privados desarrollen.

Le Boterf establecía criterios para definir una competencia esencial: a) estrategia, es decir, que sea necesaria para contribuir a materializar la política con enfoque de derechos, b) competitividad, que sea necesaria para obtener o mantener una ventaja competitiva, es decir, impedir la pérdida de demanda cautiva y, c) especificidad, que sea una construcción específica de la organización -en este caso, de los hospitales públicos autogestionados en red.

Las competencias centrales para la autogestión hospitalaria en red son indispensables para los hospitales públicos involucrados en materializar el Plan Auge. La responsabilidad última del cumplimiento de las garantías recae sobre el Fonasa, pero en una red asistencial "cerrada", la sola fiscalización de la Superintendencia de Salud sobre Fonasa no 
eliminará la lista de espera de la puerta de los hospitales.

Las competencias centrales son las siguientes:

- Desarrollo de la cartera de servicios: Esta, que es una tarea a la que necesariamente concurriría en algún momento el gestor de red -p. ej. aprobando las grandes inversiones-, es una tarea central del hospital, dinámica, que debe realizarse en función de los recursos disponibles y del desarrollo posible de las especialidades. Su frontera es la máxima capacidad resolutiva antes de pasar al nivel de atención siguiente y su piso es la máxima capacidad resolutiva del nivel anterior.

- Gestión de la referencia y la contrareferencia: Dimensión operacional de la autogestión en red y, al mismo tiempo, más específica. Se debe definir, mantener y administrar la forma y condiciones en que los pacientes son derivados a las especialidades y contraderivados para continuar sus tratamientos y controles en el origen. Esto es asunto de gran sensibilidad para los pacientes y para los tratantes, que impone una férrea disciplina de la información, del manejo de las agendas médicas, de la oportunidad de las altas, de la pertinencia en las derivaciones, de la formación y de la continuidad de los cuidados.

- Gestión de las listas de espera: Conocer y gestionar las listas de espera es indispensable para la gestión del hospital, pero implica un ordenamiento que toca distintos ámbitos del quehacer hospitalario, que deben funcionar bien y coordinadamente. Tal coordinación redundará en que las listas sean fuente de retroalimentación, se ordenen, se prioricen y se acorten $y$, al mismo tiempo, que quienes esperan reciban respuestas confiables y materializables dentro de plazos razonables.

Los hospitales autogestionados en red que gestionen bien estas tres competencias esenciales habrán conseguido operar apropiadamente en

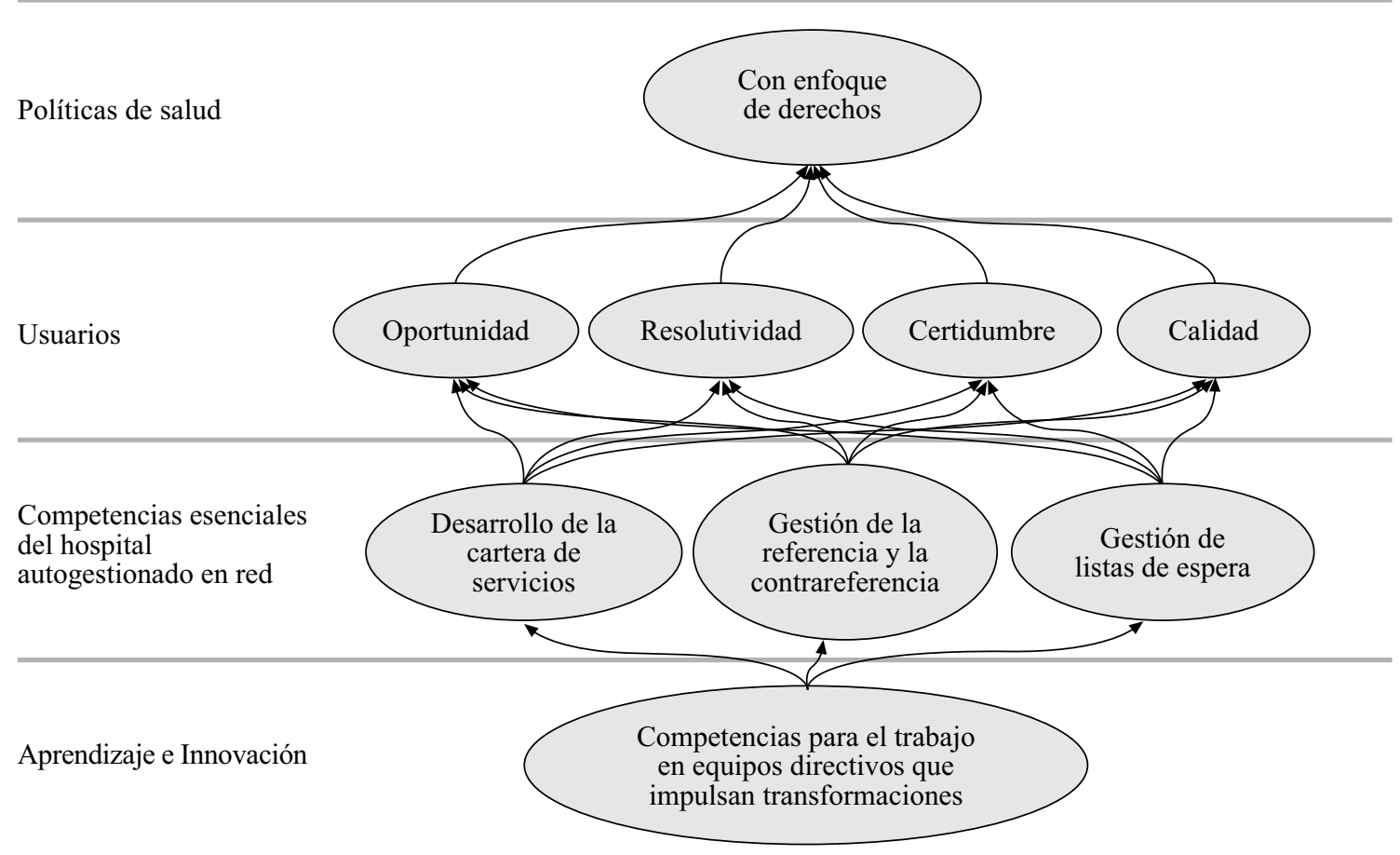

Figura 1. Mapa estratégico de un hospital autogestionado en red. 
el modelo de red asistencial "cerrada" y lo harán en la tranquilidad de que sus usuarios no migrarán en busca de alternativas porque estarán satisfechos. El buen funcionamiento de la red "cerrada" es el elemento de cambio principal frente a las restricciones para elección de prestadores y servicios.

\section{REFERENCIAS}

1. NANCUANTE, U. \& ROMERO, A. La Reforma de la salud. Instituto de Políticas Públicas y Gestión Salud y Futuro. Universidad Andrés Bello. Editorial Biblioteca Americana. 2008.

2. CUNILL, N. Las Políticas con enfoque de derechos y su incidencia en la institucionalidad pública. Revista del CLAD Reforma y Democracia. Número 46Caracas. 2010.

3. VERGARA, M. El curso de la Reforma de la Salud: el Boomerang de los excedentes. Revista Chilena de Salud Pública. 2004. 8:37-38.

4. BITRÁN, R. Public Hospitals in Chile: prospects for autonomy. Prepared for the Human Development Department of the World Bank. June 1996.

5. CLINICAS DE CHILE, A.G. Indices Financieros 2002-2008. Julio 2009.

6. PORTER, M. \& OLMSTED, E. Redefiniendo la competencia en sector salud. Harvard Bussines Review. Junio 2004.

7. MONTT, J. Enfrentando las transformaciones de los hospitales autogestionados, Capítulo III Auge y Redes Asistenciales, páginas 133 a 150. "Reforma de la salud en Chile: desafíos de la implementación". Sánchez, H. \& Labbé, J., Editores. Instituto de Políticas Públicas y Gestión Salud y Futuro. Universidad Andrés Bello. 2005.

8. GÓMEZ, P. Gestión de Redes Asistenciales. Presentación power point. 2009.

9. MINISTERIO DE SALUD. Establecimientos Autogestionados en Red (EAR). Plan de implantación de autogestión hospitalaria en red. Archivo Word. Febrero 2009.

10. DEPARTAMENTO DE DISEÑO Y DESARROLLO DE PROCESOS ASISTENCIALES (DIRED). Autogestión en Red: Proceso de Implementación. Subsecretaría de Redes Asistenciales. Presentación en power point. Marzo 2009.

11. MINTZBERG, H. Minzberg y la Dirección. Ediciones Diaz de Santos S.A., Madrid, España, 1991.

12. KAPLAN, R.; \& NORTON D. Cómo utilizar el Cuadro de Mando Integral para implementar y gestionar su Estrategia. Gestión 2000 S.A., Barcelona, 2001.

13. SHRIVASTAVA, P. Knowledge Ecosistems for Business Education and Training. httpwww.facstaff.bucknell.edu/shrivast/Knowledge Ecology.html; 1998.

14. HAMEL, G. \& PRAHALAD, G. Compitiendo por el Futuro. 1995, p.267.

15. PRAHALAD, G. \& HAMEL, G. The .Core Competence of the Corporation. Harvard Business Review, mayo-junio, 1990.

16. LE BOTERF, G. "Ingeniería de las Competencias" Ediciones Gestión 2000 S.A.; Barcelona, febrero 2001.

17. MONTT, J. Acompañamiento EAR. Subsecretaría de Redes Asistenciales, Ministerio de Salud. Presentación en power point. Agosto 2009.

Recepción: 1 de abril de 2010 Aprobación: 8 de abril de 2010

Usted puede comentar éste y otros artículos publicados en la Revista Chilena de Salud Pública, enviando un correo electrónico a revistasp@med.uchile.cl 